\title{
Modeling Surgical Procedures for Multimodal Image-Guided Neurosurgery
}

\author{
P. Jannin ${ }^{1}$, M. Raimbault ${ }^{1}$, X. Morandi ${ }^{1,2}$, and B. Gibaud ${ }^{1}$ \\ ${ }^{1}$ Laboratoire IDM, Faculté de Médecine, Université de Rennes 1, 2, avenue du Pr Léon \\ Bernard, CS 34317, 35043 RENNES Cedex France \\ \{pierre.jannin, melanie.raimbault, xavier.morandi, \\ bernard.gibaud\}@univ-rennes1.fr \\ http///idm.univ-rennesl.fr \\ ${ }^{2}$ Department of Neurosurgery, Hôpital Universitaire de Rennes, Rue H. Le Guilloux, 35033 \\ RENNES Cedex 9 France
}

\begin{abstract}
We present a model of surgical procedures that facilitates the management of multimodal information (i.e. anatomical and functional) in the context of multimodal image-guided craniotomies. We suggest that a surgical procedure be considered as a script consisting of a set of successive steps. Each step comprises a list of image entities extracted from multimodal images and relevant for the performance of this surgical step, and an action describing the purpose of the step. Each image entity has a role that may be different from step to step. This model is described by a UML class diagram and a textual description. We also present the results of a preliminary validation of the model performed on 15 clinical cases. The validation has shown the relevance of our model on the clinical cases studied and has confirmed the main assumptions we made to define the model. Even though further semantic validation is required, some benefits of this approach can be outlined: it improves management of multimodal information by describing when and why images are essential in the performance of the surgical act (e.g. place and role of images in the surgical script) and adapts visualization and interaction modes in neuronavigation. Our model should enhance preparation and guidance of surgery and lead to the development of simulation and even teaching tools.
\end{abstract}

\section{Introduction}

The development of new imaging devices providing multimodal information (e.g. anatomical and functional) with new image analysis tools (e.g. segmentation, registration and visualization) and localization systems that can match the patient in the operating room directly with his or her preoperative images has led to the growing clinical use of image-guided surgery systems (e.g. neuronavigation systems). Their intraoperative use requires a preliminary planning stage, which consists in extracting information that is relevant to the performance of the surgical act from preoperative images $[1,6,8,9]$. Information includes target areas (e.g. tumors, malformations), areas to be avoided (e.g. high risk functional areas, vessels) and reference areas (e.g. sulci, vessels, anterior and posterior commissures, basal ganglia). For example, contours of a tumor and sulci segmented from T1-weighted MR imaging, and functional data such as dipolar sources reconstructed from magnetoencephalography examination from 
motor stimulations may provide indices and references for the performance of the surgical act. Neuronavigation systems display these areas of interest during surgery as graphical overlays in a surgical microscope or as images or graphics in a companion computer workstation. Many authors have demonstrated the clinical added value of multimodal (anatomical and functional) neuronavigation [2,5,7,10,12,15].

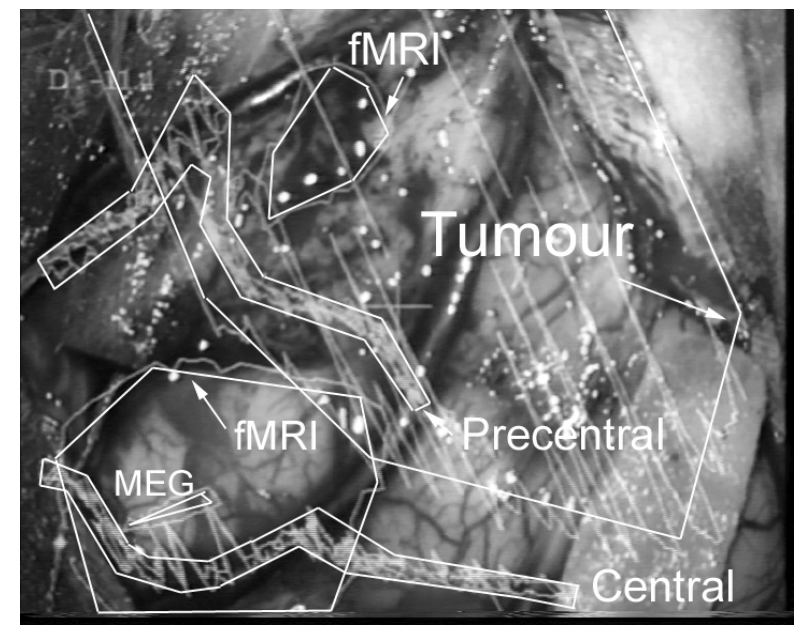

Fig. 1. Intraoperative view of graphical overlays representing anatomical information (precentral and central sulci), pathological information (oligo-astrocytoma) and functional information (MEG and fMRI for motor and somato-sensory activations) as seen through the ocular of a surgical microscope.

However, according to our experience [7], augmented reality in microscope-based multimodal neuronavigation, although it has proved to be clinically useful, is still hampered by hardware limitations (e.g. overlays are monochromic, monoscopic and consist of 2-D cut planes of 3-D surfaces) and functional limitations (e.g. the surgeon has to switch the overlays on and off to understand the graphics 1 Therefore, complex multimodal information may lead to a barely understandable scene, in which the surgeon may find it difficult to identify anatomical and functional features, as illustrated in Figure 1. This point may become crucial in exceptional cases where the person who did the planning is not the one actually performing surgery, although this may occur increasingly often, or if the surgical strategy has to be discussed between people located in different places. This paper focuses on the management of multimodal information in neuronavigation systems. We suggest that the management of multimodal information can be improved based on prior knowledge of surgical procedures. This includes two aspects: a «data management» aspect, enhancing when and why images are essential in the performance of the surgical act (e.g. place and role of images in the surgical script) and a "representation and interaction »

1 Of course, another major issue of image-guided surgery is the possibility to update preoperative images according to intraoperative anatomical modifications due to the surgical procedure. 
aspect focusing on selecting appropriate visualization and interaction features for image entities, according to their place and role in the surgical script.

More precisely, this paper introduces a model of surgical procedures that breaks them down into steps and associates a goal and relevant information with each step. The object of this research was not to define the surgical procedure with its every detail (e.g. taking into account surgical tools and bio-mechanical tissue properties) as it may be done for robotic purposes. Our primary concern was rather to manage multimodal information more ingeniously to improve and simplify multimodal imageguided craniotomies, especially when using microscope-based multimodal neuronavigation systems. We chose to build and validate our model with three kinds of surgical procedures in which multimodal image-guided surgery provides a real added value: surgery of supratentorial intra-parenchymatous tumors, surgery of supratentorial cavernomas, and selective amygdalo-hippocampectomy for medically intractable epilepsies. We present the results of a preliminary validation of the model performed on 15 clinical cases.

\section{Methods}

The basic idea was to break down the surgical procedure into steps defining the surgical script. Steps can be determined during the planning stage of the surgical procedure. A list of relevant image entities is assigned to each step (i.e. anatomical and/or functional entities extracted from preoperative images) and the role of each entity in the step is specified (i.e. target area, area to be avoided, reference area). One of our initial goals was to adapt interaction and visualization features according to this model. For instance, during surgery, only the relevant image entities belonging to the step currently being performed are displayed, and colors are related to their role. That way the neurosurgeon can focus his or her attention on the basic information of a given step of the surgical procedure.

We defined a generic information model describing the main concepts and relationships involved in our field of interest (i.e. multimodal image-guided craniotomies). We chose UML [11] (Unified Modeling Language) among the numerous techniques and formalisms available because it may now be considered as a standard. To produce a generic model, we studied three types of procedures, accounting for approximately $75 \%$ of supratentorial procedures in our neurosurgical department (excluding traumatology, shunts, and vascular procedures): surgery for supratentorial intra-parenchymatous tumors (67\%), for supratentorial cavernomas $(5 \%)$ and selective amygdalo-hippocampectomies for medically intractable epilepsies (3\%). We selected these procedures because they can benefit significantly from multimodal neuronavigation. The design of the model started with the analysis of surgical procedures as described by neurosurgeons in both generic descriptions (i.e. referring to standardized procedures expressed as an abstraction of many concrete clinical cases) and specific clinical cases studied both during surgery and postoperatively from videotapes (6 tumours, 3 cavernomas and 3 amygdalohippocampectomies). This analysis was then formalized into UML object diagrams, describing instances of objects involved, and a UML class diagram, describing the corresponding classes. The final class diagram was iteratively obtained by assessing 
the model with the three types of procedures. We used a UML design tool (Visual UML 2.5, Boulder CO, USA) to design the model and validate the syntax of the scheme.

In order to demonstrate the relevance and the added value of this approach, we developed a software application to instantiate the model in clinical situations. The neurosurgeon defines a number of surgical steps and assigns to each of them relevant image entities selected from a list of available 3-D image entities previously segmented from preoperative data. Anatomical information may include skin and brain surfaces, ventricles, sulci, and lesions segmented from 3-D T1-weighted anatomical MRI and vessels segmented from MR Angiography. Functional information may include volumes of interest extracted from correlation volumes computed from functional MRI examinations, equivalent current dipoles reconstructed from MEG and epileptogenic areas segmented from ictal SPECT. A role is attributed to each image entity. Display parameters (color and transparency) can be independently assigned to each image entity associated with a surgical step. A 3-D VRML scene is generated for each step, including 3-D surfaces of the corresponding image entities. A viewpoint and a view direction (i.e. view trajectory) can be assigned to each scene. Display parameters and view features are stored along with the 3-D surfaces of the image entities in each VRML file. The surgeon can therefore visualize the procedure step by step by displaying the different 3-D scenes corresponding to the successive steps.

\section{Results}

Figure 2 presents a UML class diagram showing the major conceptual entities involved in our field of interest and their relations.

SurgicalProcedure is the basic entity of this model. A SurgicalProcedure concerns one or more Targets and comprises one or more ordered Steps. In multimodal imageguided surgery, the procedure may require several ImageEntities corresponding to image entities (points, surfaces or volumes) extracted from multimodal images (anatomical or functional). A Target may have an ImageEntity which is its graphical representation segmented from images. A Target has properties (e.g. size, orientation and amplitude) and is located within the right and/or left hemisphere (Side). A Target may concern several anatomical concepts (AnatConcept) such as a gyrus or a lobe in which it is located. A Target is also characterized by a pathological concept (PathoConcept) such as a cavernoma, a glioma or an epileptogenic focus. A Concept is an abstract class presenting the properties that are common to all of its specializations (PathoConcept, AnatConcept, FunctConcept). It may eventually refer to existing knowledge of these concepts through terminology such as Neuronames [3] included in UMLS since 1992. Each ImageEntity refers to one or more Concepts, representing anatomical, functional or pathological information.

A Step comprises a single Action, which is the goal of this step (e.g. incision of the dura mater). An Action acts upon one pathological, functional or anatomical entity (Concept). A Step may also have an ImageEntityList, listing the ImageEntities (anatomical and/or functional) relevant to this specific Step. Each ImageEntity belonging to a specific ImageEntityList has a Role representing the kind of use 
anticipated for that instance in that Step, such as target, area to be avoided, or reference areas. A Step may refer to an ActionModel and may be described by one or more ActionAttributes. An ActionModel may be for instance a graphical element representing the action to be performed, such as contours of the craniotomy or a segment representing an approach to a cavernoma. An ActionAttribute provides further details on the action to be completed. For example, the action of positioning the patient may be further described by the position to be used (right or left lateral positions, supine or prone positions).

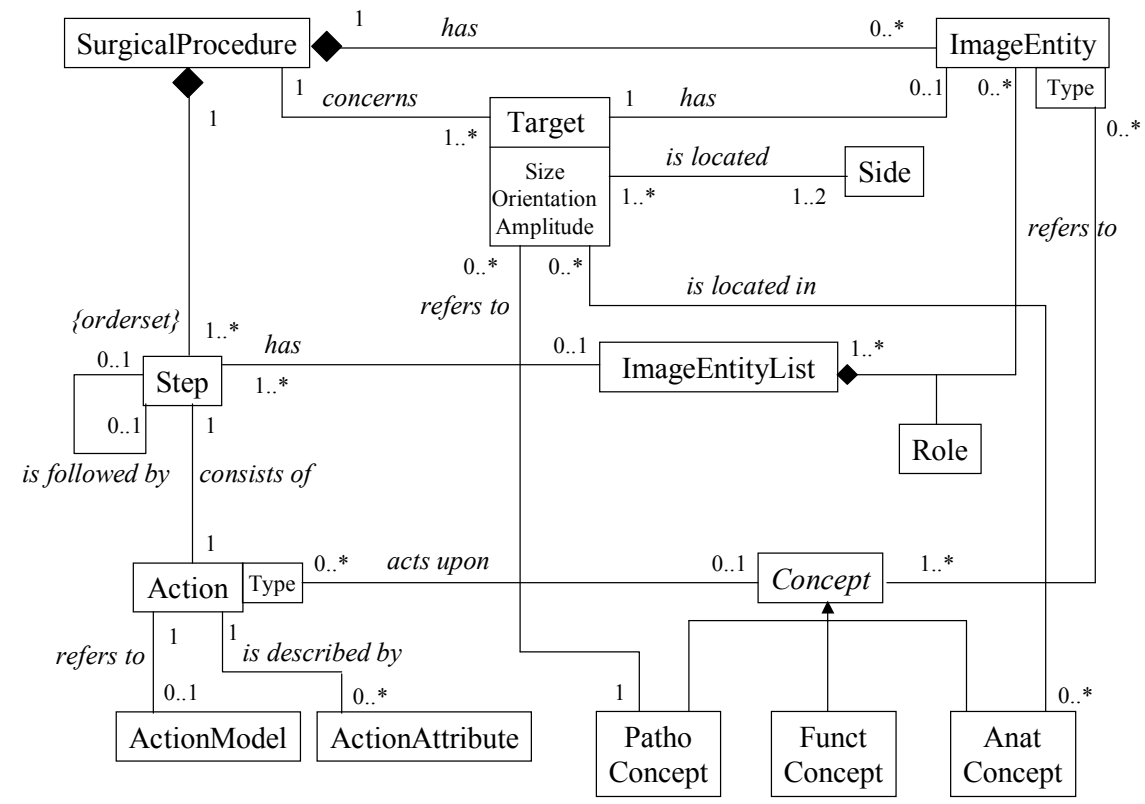

Fig. 2. UML class diagram of multimodal image-guided craniotomies

We instantiated our model with our software application for a few clinical cases, and verified that the 3-D display modes and the production of one 3-D virtual scene per surgical step - including the relevant image entities, viewpoint and a single trajectory - were well adapted to our requirements.

\section{Validation}

\subsection{Validation Method}

The semantics of the model were validated with a retrospective study. Forms listing the objects of our model were designed and filled in by three different neurosurgeons with the instances of the objects relative to real surgical procedures. Clinical cases 
were selected according to the scope of the model (9 supratentorial intraparenchymatous tumors, 3 supratentorial cavernomas, and 3 selective amygdalohippocampectomies for medically intractable epilepsies). Naturally, these clinical cases were different from those used to design the model. The operator could define as many steps as required for the procedure and as many ImageEntities as ideally required for the action implemented in each step (i.e. what kind of image information should the graphical overlay display in the oculars of a surgical microscope to perform surgery?). Surgeons were relatively free to fill in forms with any values. But for some classes (e.g. Role, Side) we provided a list of possible values. For instance for the Role class, they had the choice between "target", "reference" or "to be avoided" values. We also asked the surgeons to underline and document situations where the model could not fit the clinical requirements.

\subsection{Validation Results}

Globally, the model was validated. Surgeons who filled in the forms - but did not participate in designing the model - were enthusiastic about this approach. Surgeons all confirmed that they do not need to see all of the image entities at every step and that the model helps manage them. Results of validation do not question the model but raise problems related to specific relation cardinalities between the classes and values used to instantiate the classes.

One of our main assumptions was that a surgical procedure could be broken down into separate steps. We defined a step as the realization of a single action, even though the performance of two successive actions may require the same ImageEntityList. This assumption was confirmed, but surgeons noted that it was not always trivial to know how detailed one should be in defining an action and therefore where one step begins and finishes. They highlighted the importance of the ActionAttribute class to characterize surgical tools or type of action (e.g. skin incision).

We constrained the action to act upon no more than one Concept. In two particular cases we realized that this cardinality had to be extended to one Concept or more. For a right-handed patient with a right temporal glioblastoma, the resection step concerned both tumor and adjacent cerebral tissue (partial temporal lobectomy) and consequently the Step acted upon two Concepts. One difficulty when filling the forms was related to the instance values to be used. For the ActionModel class, which concerns a graphical representation of the action, it was sometimes difficult to find a representation that was not an image entity and to differentiate the target from the action. For the resection of a deep cavernoma, the surgeon chose a trans-sulcal approach via the pre-central sulcus. Therefore the set of values proposed for the Role class has to be extended to take into account the "trajectory" value. A last remark concerns the Concept class and related PathoConcept, FuncConcept and AnatConcept classes. It was not easy for the surgeon to understand exactly what kind of information these concept classes involved and make the difference between information directly related to the patient (e.g. exact anatomical location of an image entity) and generic knowledge (e.g. the precentral gyrus is an anatomical part of the frontal lobe). Therefore, it may be relevant to distinguish two levels: one for instantiated knowledge and a second for canonical knowledge [14]. 


\section{Discussion and Conclusions}

In this paper we introduced a generic model of neurosurgical procedures to improve multimodal image-guided craniotomies. Few studies have described surgical procedures models. The closest existing work provides a common terminology of the surgical procedures names (the GALEN/GASP representation $[13,17]$ ) but without considering its dynamic aspect. Our model manages image information according to its place and role within the surgical script. During neuronavigation, it may contribute to adapt visualization and interaction modes. This model provides a framework to model knowledge involved in the performance of neurosurgical acts and therefore leads to the definition of an ontology 4] of our field of knowledge. In this approach, semantics (i.e. meaning) are assigned to image data (i.e. image entities) relatively to our context (multimodal image-guided craniotomies). Making this meaning explicit is a key aspect of successful information sharing between the different software components used for planning and surgery and between which a perfect interoperability is required.

Validation has shown the relevance of our model on the clinical cases studied, confirmed our main assumptions and provided us with some leads to improve our model (e.g. modifying some cardinalities, refining possible values for classes, adding new features to the Target class, distinguishing instantiated knowledge from canonical knowledge). However, this validation has to be improved by involving a broader set of clinical cases and surgical procedures and a larger community of neurosurgeons.

The study of the different clinical cases emphasized the fact that there are few variations between cases requiring the same surgical procedure. The same steps are associated with the same actions and similar relevant image entities. Consequently, it may be worth defining models of these specific procedures, which would a priori include the expected steps and the image entities to assign.

This paper presents a work in progress. While further validation and modifications are required, the first benefits of this approach can already be outlined. It should confer real added value to the different levels of image-guided surgery, from preprocessing to planning, as well as during surgery. Models of surgical procedures can manage image data according to the surgical script, which should lead to better anticipation of surgery through the development of simulation tools. Furthermore, they may greatly improve the performance of surgery under microscope-based neuronavigation systems by optimizing both visualization and interaction features of multimodal preoperative images. Finally our approach may facilitate the decisionmaking process (e.g. choice between surgery, biopsy or radiotherapy), and may contribute to generate case reports and develop teaching tools.

We are convinced that our approach, in conjunction with other a priori knowledge such as anatomical and physiological models [16], will contribute to defining decision support systems to improve image-guided surgery. 


\section{Acknowledgements}

We wish to thank E. Seigneuret and L. Riffaud from the Neurosurgical Department of the Hôpital Universitaire of Rennes (France) who participated in the design and validation of the model. We would also like to thank O. Dameron, from the Laboratoire IDM, for fruitful discussions to improve the model and Pr. J.M. Scarabin, from the Laboratoire IDM and the Neurosurgical Department, Rennes. This research program was supported in part by grants from the "Conseil Régional de Bretagne".

\section{References}

1. Apuzzo MJ: New dimensions of neurosurgery in the realm of high technology: possibilities, practicalities, realities. Neurosurgery, 38 (1996) 625-639

2. Alberstone CD, Skirboll SL, Benzel EC, et al.: Magnetic source imaging and brain surgery: presurgical and intraoperative planning in 26 patients. J Neurosurg 92 (2000) 79-90

3. Bowden DM, Martin RF: Neuronames Brain Hierarchy. Neuroimage, 2 (1995) 63-83

4. Chandrasekaran B, Josephson JR, and Benjamins VR: What are ontologies and why do we need them? IEEE Intelligent Systems January-February (1999) 20-26

5. Gallen CC, Bucholz R, Sobel DF: Intracranial neurosurgery guided by functional imaging. Surg Neurol, 42 (1994) 523-530

6. Hardenack M, Bucher N, Falk A, and Harders A: Preoperative planning and intraoperative navigation : status quo and perspectives. Computer Aided Surgery 3 (1998) 153-158

7. Jannin P, Fleig OJ, Seigneuret E, et al.: A data fusion environment for multimodal and multi-informational neuronavigation. Computer Aided Surgery 5 (2000) 1-10

8. Kikinis R, P. Langham Gleason, Moriarty TM, et al.: Computer-assisted threedimensional planning for neurosurgical procedures. Neurosurgery 38 (1996) 640-651

9. Kockro RA, Serra L, Tseng-Tsai Y, et al.: Planning and simulation of neurosurgery in a virtual reality environment. Neurosurgery 46 (2000) 118-137

10. Maldjian JA, Schulder M, Liu WC, et al: Intraoperative functional MRI using a realtime neurosurgical navigation system. J Comput Assist Tomogr 21 (1997) 910-912

11. Muller PA. Modélisation objet avec UML. Eyrolles Eds (1999)

12. Nimsky C, Ganslandt $\mathrm{O}$, Kober $\mathrm{H}$, et al: Integration of functional magnetic resonance imaging supported by magnetoencephalography in functional neuronavigation. Neurosurgery 44 (1999) 1249-1256

13. Rector AL, Rogers JE, and Pole P: The GALEN High Level Ontology. Proceedings of Medical Informatics in Europe (MIE'96), IOS Press, Amsterdam (1996) 174-178

14. Rosse C, Mejino JL, Modayur BR, et al.: Motivation and Organizational Principles for Anatomical Knowledge Representation. JAMIA 5 (1998) 17-40

15. Schulder M, Maldjian JA, Liu WC, et al: Functional image-guided surgery of intracranial tumors in or near the sensorimotor cortex. J Neurosurg 89 (1998) 412418

16. Taylor C, Draney MT, Ku JP, et al.: Predictive medicine: computational techniques in therapeutic decision making. Computer Aided Surgery 4 (1999) 231-247

17. Trombert-Paviot B, Rodrigues JM, Rogers JE, et al.: GALEN: a third generation terminology tool to support a multipurpose national coding system for surgical procedures. International Journal of Medical Informatics 58-59 (2000) 71-85 